\title{
PAPULAR URTICARIA
}

\author{
BY \\ ARTHUR ROOK and W. FRAIN-BELL \\ From the Department of Dermatology, United Cardiff Hospitals
}

(RECEIVED FOR PLBLICATION APRIL 24, 1953)

Papular urticaria is one of the commonest and most troublesome skin diseases of early childhood. The controversy as to whether the eruption is of parasitic origin or whether it is related to digestive disturbances, allergic or otherwise. is now approaching its centenary and is still vigorously debated. The inconsistent response to our own therapeutic efforts prompted us to study a series of cases in some detail and to review the literature which, for so common a condition, is surprisingly scanty.

\section{Clinical Description}

Thomas Bateman in 1813 wrote:

'There is scarcely any limit to the varieties of these papular affections, but I have observed one form which is so uniform in its character as to be entitled to notice here. It might be called lichen urticatus: its first appearance is in irregular inflamed wheals, so closely resembling the spots excited by the bites of bugs or gnats as almost to deceive the observer. The inflammation, however, subsides in a day or two leaving small, elevated, itching papules. While the first wheals are thus terminating new ones continue to appear in succession until the whole body and limbs are spotted with papules which become bere and there confluent in small patches. The eruption is peculiar to children: it commences in some cases soon after birth and sometimes later and continues with great obstinacy for many months. Both the wheals and the papules are accompanied by intense itching, which is exceedingly severe in the night, occasioning an almost total interruption of sleep and a considerable loss of flesh.'

Although later observers have added to our knowledge of the natural history of the condition, this clinical description is still among the best available.

Willan and his pupil Bateman made an effort to place the morphological description of skin lesions on a sound basis, but for the greater part of the nineteenth century the nomenclature of the papular eruptions of infancy and childhood remained confused. The same condition is often described under several different terms and the same term has been applied by various authors to apparently different conditions. By the end of the century greater uniformity of nomenclature had been achieved and the terms still employed by most British and American writers, papular urticaria and lichen urticatus, were regarded as synonyms. Strophulus is now generally regarded as synonymous with papular urticaria but even this name has been employed for other papular eruptions. In France strophulus or acute simple prurigo of Brocq are the terms in common use.

Jonathan Hutchinson (1879) emphasized the greatly increased frequency of papular urticaria in the summer months. Of 109 cases, 82 were seen between May and October, 27 between November and April. Adamson (1907) also noted that the peak incidence was between July and September. $\mathrm{He}$ found the sites most frequently affected to be the lumbar region, the buttocks, the outer sides of the arms and thighs and the wrists and hands. The papules succeeding the wheals are often surmounted by small vesicles and occasionally bullous lesions develop. Adamson noted the greater rarity of the severe forms in his better class patients. He gives the duration of the condition as a few days to two or three years.

Barber (1914) in an excellent clinical description emphasizes the tendency, which other authors had previously noted, for the lesions to appear in groups of six to 20 papules at intervals of two or three days. Tomkinson (1926) reporting on 102 cases seen in Glasgow merely confirmed earlier observations and noted that the sex incidence was equal.

Hallam (1927) published a careful study of 52 cases. The minimum age of onset was 3 weeks and the maximum 3 years, the average age at onset being just under 1 year. The eldest patient was aged 13 and had had the condition since the age of 1 . Thirty-nine began in the summer months but 21 of these also had the eruption at times during the winter. The sites most favoured by the eruption were the buttocks, the lower third of the back, the thighs, ankles and forearms. In only two instances were two cases present in the same family. Thirty-one hospital admissions all resulted in rapid fading of 
the eruption and no new lesions developed while the children were in hospital. The onset of the eruption in four cases coincided with removal to a new residence. One child was twice cleared of the eruption while on holiday. Corson, Perlman, Waltz and Ash (1931) investigating 31 cases in Philadelphia confirmed in general the work of other writers but did not agree in considering involvement of the face to be uncommon. Hallam (1932) published further clinical investigations. Eleven children were admitted to hospital for the nights only, spending the day at home. In eight no new lesions developed and in three cases a few sporadic papules appeared. Keeping the children in hospital by day only had no beneficial effect. Bringing the bedding from home to hospital led to the appearance of a few papules in one case on one occasion only. Hallam collected the incidence figures for papular urticaria in children under 14 in eight dermatological out-patient departments in London and provincial hospitals for the years 1927 to 1930 . The figures ranged from $0.8 \%$ to $24 \%$ of out-patient attendances, the three London hospitals showing a notably high incidence. The annual incidence often showed striking variation from year to year, and in all the hospitals the incidence was exceptionally high in 1930.

Among Gordon's (1933) 50 cases more than one member of the family was affected in six instances. Onset or recurrence of the condition was noted to occur on returning home from hospital in 10 cases. Kinnear (1933) recorded the development of papular urticaria in 14 children aged 7 months to 9 years on arriving in this country from America. He confirmed that the eruption disappears when the child is admitted to hospital.

Pillsbury and Sternberg (1937) investigated 60 cases. They found that $80 \%$ developed below the age of 3. The sites involved in order of frequency were extensor forearms, the anterior surface of the upper arms and the back. Bray's (1937) observations on 200 cases agree in general but Bray considered that the face and other exposed parts were rarely involved.

\section{Aetiology}

The many aetiological hypotheses which have at one time or another found supporters can conveniently be considered under four main headings.

Digestive Disturbances and Food Allergy. Many of the earlier writers on papular urticaria attempted to relate the condition to digestive disturbances of various types and to dentition. As the concept of allergy developed during the first two decades of the present century the older theories were reclothed in new jargon and many investigations were undertaken employing new technical procedures.
Radcliffe Crocker (1903) believed that irritation of the alimentary canal played an important role but Adamson (1907) was not satisfied with the current theory that digestive disturbances were responsible.

The authors who consider food allergy to be the cause of papular urticaria base their beliefs either on the results of skin testing or on the improvement of the condition on suppressing the foods considered to be responsible. Sidlick and Knowles (1922), using intradermal tests, found that nine of 12 children with papular urticaria reacted to one or more proteins. Three were cured when the incriminated foods were suppressed, four were improved and two were unimproved. This investigation was uncontrolled and it is not stated whether any of the children was admitted to hospital and, if so, whether readministration of the food in hospital reproduced the eruption. Corson et al. (1931) studied 31 cases and noted that the parents of the children were eager to incriminate foods in 23 cases. All skin tests were negative in 21 of the patients. In only one did elimination and readministration of a food substance apparently influence the eruption.

Walzer and Grolnick (1934) in a similar investigation found positive skin tests to one or more allergens in only nine of 36 cases of papular urticaria. Specific therapy as suggested by the skin tests was of no value. These authors consider papular urticaria to be an atopic disorder.

Neither Hallam (1927) nor Gordon (1933) was able to establish a dietary factor in any of their patients.

Pillsbury and Sternberg (1937) considered that the evidence in support of an allergic origin of papular urticaria was meagre. Bray (1937), however, strongly supported the relationship to food allergy. $\mathrm{He}$ considered that fats, especially pig products. fish, eggs and chocolate were frequently responsible, and he claimed to have produced new lesions within 24 hours of feeding a child with pork, ham or bacon, and suggested that the coexistence of a thermal factor was necessary to produce the skin lesions, and attempted to explain in this way the seasonal incidence and the frequent development of new lesions at night.

Tate (1935) investigated very thoroughly a series of cases of papular urticaria and gave a valuable review of the literature. He was unable to establish food sensitivity as a cause in any single case. He confirmed the observations of Hallam and others that the aetiological agent was connected with the patient's home environment and that it was not bedding or clothing although it could be carried on these articles. The incidence of a family history of 
atopic disorders was no higher in his cases than in normal controls.

Urbach (1938) claimed that sensitivity to a food protein or occasionally a carbohydrate substance could be established as the cause of the eruption in $28 \%$ of his 225 cases.

Although food allergy is still widely accepted as a cause of papular urticaria it is impressive that there is no published series of cases that we can discover, with the possible exception of Urbach's, in which it has been claimed that administration of any food to a child in hospital under controlled conditions has ever provoked papular urticaria. Many of those who have investigated the problem most thoroughly have been able to convince themselves that food sensitivity plays no part whatever in this disease. The seasonal incidence, the striking variation in frequency from year to year and the complete immunity of the child in hospital cannot be accounted for on the basis of food allergy.

Nervous Factors. Gordon (1933) was impressed by the importance of psychological factors. Other authors have also raised this question but it has received little consideration in recent years. Winnicott (1934) considered that papular urticaria was a normal phenomenon of an erectile organ, a reaction to nervous stress or to external causes. Nervous stress can apparently heighten the reactivity of the skin but there is as yet no evidence that nervous factors play any part in papular urticaria.

Infection. Botter (1952) prepared a Seitz filtrate of the nasopharyngeal washings of four mothers whose children had papular urticaria. He dropped the filtrate into the nasopharynx of eight children who had recovered from papular urticaria and of one child with eczema. All the children developed an eruption 'resembling papular urticaria although the efflorescences were somewhat smaller, sometimes grouped and not as intensely itching as in the typical disease'. No virus could be demonstrated. This work obviously requires confirmation. If an infective agent is responsible for papular urticaria it is difficult to account for the complete lack of reports of contact cases when these children are admitted to hospital.

Parasites. Hutchinson (1879) wrote:

-Is it not very singular that one author after another who has looked carefully at the spots has been puzzled to tell whether they were bites or not? ... My belief is that in very many cases the eruption is simply the result of flea bites on a pruriginous skin. ... It is the fact that only in a few persons does the flea bite cause a wheal which misleads the nurse.'

Radcliffe Crocker (1903) wrote:

'Hutchinson considers this disease to be entirely due to flea and bug bites and the like in the first instance. I am convinced this is far too narrow a view and that, though true of many cases, among the poor especially, irritation of the alimentary canal plays quite as, or even more, important a role in children than in adults, to say nothing of the recognized causes of urticaria.'

The main objections raised to the parasitic origin of papular urticaria have been the relative infrequency with which more than one member of a family has been affected in most series of cases, the fact that new lesions did not develop when children slept in their own clothing and bedding in hospital (Hallam), and the apparent improbability of a parasitic origin in certain cases. Many authors have insisted on their own ability to differentiate insect bites from 'true papular urticaria'.

The parasitic theory had had few supporters in the two previous decades when Hamburger and Dietrich (1938) claimed that all or most cases of papular urticaria were due to insect bites. Many children are not sensitive to bed bug bites, but about a third develop a wheal and later a bulla if exposed to the bite. Two-thirds to three-quarters of children with papular urticaria, however, are sensitive to bed bug bites and the evolution of the bites is similar to the lesions of papular urticaria. Further investigations (Dietrich, 1938) showed that two-thirds of 50 children with papular urticaria were sensitive to bed bug bites and the remainder to flea bites. All cases were sensitive to one or the other and some were sensitive to both. Blank, Shaffer, Spencer and Marsh (1950) and Shaffer (1950) tested 30 children with papular urticaria with flea antigen and bed bug (cimex) antigen. Seventy-seven per cent. were sensitive to one or both, whereas only two of 124 controls were sensitive. Among 87 cases there was known exposure to fleas in 26 and to bed bugs in eight and possible exposure in 72 . Eighty-eight cases were treated by conventional methods. Twenty-one per cent. were cured in an average of ten weeks. Fifty-four cases were treated with $5 \%$ D.D.T. in talc used as a dusting powder and their quarters were sprayed with 'flit' containing $5 \%$ D.D.T. Eighty-six per cent. were cured in two to three weeks. Blank and Shaffer point out that all the observed features of the natural history of papular urticaria are explained if the insect hypothesis is accepted.

Tate was one of the first to draw attention to the fact that the papular lesion is essentially different from true urticaria. A patient of his with papular urticaria developed a true urticaria due to fish sensitivity, the lesions consisting only of evanescent wheals. Histamine phosphate, 1 in 1,000 , pricked into the skin of a child with papular urticaria produced only a transitory wheal as in normal 
controls. Small pieces of nettle also produced whealing without papule formation. Cornbleet (1949) emphasized that the failure of papular urticaria to respond to antihistamines was further evidence that the reaction is of a special type. The observed lesions of papular urticaria with their biphasic course, initially urticarial and later papular, conform exactly with the known reaction to insect bites as established by Mellanby. Mellanby (1946) working with the yellow fever mosquito was able to show that subjects bitten for the first time develop an early erythematous macule followed after 24 to 48 hours by a papule. After repeated bites the immediate reaction was an urticarial wheal followed by a delayed papule. Later the delayed reaction disappeared and there was less itching and eventually, with complete desensitization, even the immediate reaction failed to appear. The length of time required for complete desensitization varied considerably from one individual to another. Rockwell and Johnson (1952) confirmed Mellanby's findings. They also were working with mosquitoes. Two of their patients had been diagnosed as papular urticaria by dermatologists.

These important observations not only offer an explanation for the type of lesion observed in papular urticaria but also explain such observations as those of Kinnear, already quoted, and other observations, including our own, in which individuals exposed to a new environment develop papular urticaria. Gordon (1950) showed experimentally that some individuals develop no reaction to insect bites. Taken in conjunction with Mellanby's work Gordon's results account both for the fact that only a small proportion of those exposed to the insects may develop papular urticaria and for the spontaneous cure of the condition after a variable duration in the majority of cases. The occasional persistence of papular urticaria into adult life is accounted for by the occasional failure of desensitization as noted by Mellanby. Shaffer, Jacobson and Pori (1952) consider that the evidence in favour of insect bites as the cause of papular urticaria is strong as it is based on the combined results of studies on different aspects of the problem, clinical epidemiological, allergic (skin tests), histological and therapeutic. The similarity of the course of experimental sensitivity to insects and natural papular urticaria is striking.

Life Histories of Fleas and Bed Bugs. Many of the difficulties which some authors have felt in accepting the parasitic aetiology of papular urticaria are readily explained when the life histories of fleas and bed bugs are studied (Herms, 1950). The eggs of the human flea. Pulex irritans, and the cat and dog fleas, Ctenocephalides felis and canis, are laid in the nests or sleeping quarters of their hosts. They are dry and do not remain attached to the hosts hairs. Eggs are usually laid in warm weather, particularly when the humidity is high, and the incubation period, which varies from two to 12 days, is shortest under these optimum conditions. The larval stage lasts from nine to over 200 days depending on whether conditions are favourable. The larvae can be found in the crevices of floors and under carpets or in kennels. They can survive with little or no food. The length of the pupal period also varies with the temperature and is between seven and 300 days. The adult fleas visit the host only for short periods for feeding and can remain for many months without food. The host specificity of fleas is not well marked and these three species will all freely bite man. The fact that fleas visit their hosts only for a few minutes at intervals of two or three days in order to feed explains both the failure of Hallam's experiments with clothing and bedding and also the liability for new lesions of papular urticaria to develop at night and at short, irregular intervals. The maximum incidence of papular urticaria in the warm, moist months of the year coincides with the season of maximum prevalence of fleas.

The bed bug, Cimex lenticularis, lays its eggs on rough surfaces, in cracks and crevices and behind woodwork or wallpaper. The eggs are attached by a quick drying cement and may therefore require stiff brushing to remove them. In warm weather the adult may lay two to three eggs daily but in spring and autumn, when the temperature is lower. the frequency of egg laying is considerably reduced. The eggs do not survive longer than three months in uninhabited houses. After hatching the nymph can live for three or four months at low temperatures without food but further development cannot occur. If food is available the nymph moults five times before reaching full adult development. An average time of 10 weeks is required from egg to adult.

The adult bed bug is a flat, oval insect one-fifth of an inch long and one-eighth of an inch broad. Its colour may be light or dark brown but after feeding is usually purple or red. It is chiefly nocturnal but will feed in the day time if conditions are favourable, and is said to be most active just before dawn. Under ideal conditions of temperature feeding takes place about once a week but in colder weather the frequency is reduced. The time required for feeding varies from five to 12 minutes after which the insect leaves its victim as fast as possible. The adult bed bug has been known to survive a year without feeding and for as long as four years with 
occasional feeds. The temperature of the house is likely to have a considerable effect on the breeding of the bugs. If the temperature never falls below $55^{*} \mathrm{~F}$. for more than a few hours during the winter as, for example, in a bed-sitting-room, feeding and breeding will continue throughout the year. If the room is without artificial heat breeding will cease and feeding will become very infrequent during the winter. The bed bug is capable of travelling long distances in search of food, even from one house to another. The temporary storage of furniture, the purchase of secondhand furniture or its carriage in an infected van are all potential sources of infestation. The percentage of houses infested with bed bugs is highest in large cities, and, despite the efficacy of modern methods of control, bed bug infestation is still common.

\section{Personal Cases}

For the purpose of this article we have studied a series of 100 consecutive cases. The sexes were equally affected. The minimum age of onset was two weeks (papular urticaria developed within 24 hours of returning from the maternity home) and the maximum seven years; the average age of onset was 1 year and 10 months, $89 \%$ of our cases being under 3. Reliable information regarding seasonal incidence was available in 61 cases. Of these $62.3 \%$ were affected more severely in the summer and $9.2 \%$ in the winter. The remaining $29.5 \%$ were equally affected throughout the year. The distribution of the lesions was accurately recorded in 75 cases. In the majority the legs and the lower trunk were principally involved. The face was involved in $5.8 \%$. All these findings are in general agreement with those of other authors. Some authors have, however, been impressed by the rarity with which other members of a family were similarly affected. Special inquiry on this point was made in 85 cases and in no fewer than $31.7 \%$ was another member of the family similarly affected. Since $21 \%$ of these cases were in only children, approximately $50 \%$ of the families in which there were other children at risk had one or more of these affected. In many families the children were affected in succession, rather than simultaneously, and frequently older siblings were stated to have suffered from 'heat bumps' at about the same age as the patient. In many families, however, the children were affected simultaneously.

In all cases the characteristic irregular grouping of the papules was noted and in several cases the lesions were grouped above or below the sites of pressure of elastic or other constrictions of underclothing or pyjamas, the actual site of constriction being free. The eruption was sometimes profuse and involved the greater part of the trunk and limbs conforming precisely with the classical conception of severe papular urticaria. In a few cases only two or three groups of papules were present and the diagnosis of insect bites would have been accepted without hesitation. Often a circumscribed and a profuse eruption were seen in different members of a family at the same time or in the same child at different times. We were able to confirm the constancy with which the lesions disappear when the child is admitted to hospital. The child remains clear while in hospital and quite regularly presents himself at the out-patient department with a new crop of lesions within a short time of returning home.

Blank et al. (1950) tried to assess the percentage of the children exposed to fleas or bed bugs. After careful study of the life histories of these insects we came to the conclusion that it is very seldom possible completely to exclude exposure to one or other of them. There were pets (cats or dogs) in the homes of 43 of the 51 children whose parents were specifically questioned on this point, but these figures have little value as no control figures are available. Moreover, many other parents mentioned that reighbours ${ }^{\circ}$ pets were frequently in the house. Bed bug infestation is still relatively common in urban areas. In many cases, including some of those we have cited, the time of onset of the urticaria coincided too closely with the arrival of a new pet for this source of infestation to be ignored, but in the majority of cases precise data were almost impossible to obtain and unless the child is actually in hospital exposure to parasites can rarely be excluded with certainty.

We experienced similar difficulty in assessing the response to treatment. In some cases the response to the use of $5 \%$ D.D.T. in talc as a dusting powder was quite dramatic. In others dusting powder produced only a partial cure and in a few it was of no benefit. In some of the latter we were able to establish that the patients did not carry out our instructions. In some cases the removal or treatment of pets produced an immediate cure. The following cases are representative of the whole series:

Case 10. A boy aged $2 \frac{1}{2}$ years developed papular urticaria at the age of 18 months, his sister being first affected at the same time. Both children cleared simultaneously when their family moved from the home of an aunt with whom they had been living since the onset of the condition.

Case 12. A small boy aged $2 \frac{1}{2}$ together with his sister suffered from attacks of papular urticaria from October, 1951, until January, 1952. When the children were last seen in August, 1952, neither had had any new lesions since January. No treatment had been given and no dietary restrictions imposed, the only variable factor 
being the presence of the cat which was purchased in October, 1951, was lost in January, 1952, and did not return.

Case 15. A girl aged 3, had lived in Durham up to the age of 18 months. She developed urticaria within a few weeks of the family moving to Cardiff and attacks have occurred at intervals since.

Case 20. A boy aged $2 \frac{1}{2}$ had been living in Birmingham until two weeks before he was first seen. The day after his arrival in Cardiff he developed extensive papular urticaria which became secondarily infected. He was admitted to hospital and developed no fresh lesions. During the time he was in hospital his sister continued to attend the out-patient department with repeated crops of new lesions. This boy had had a similar short attack whilst staying with his grandmother in Cardiff at the age of 6 months. It had cleared immediately when he returned to his home in Birmingham.

Case 29. A small girl aged 5, together with her brother, suffered from attacks of papular urticaria which regularly coincided with visits to her grandmother. The attacks started at the age of 1 . Another grandchild, usually living in Germany and never previously affected, also developed papular urticaria whilst spending a holiday with the grandmother.

Case 41. A brother and sister aged $2 \frac{1}{2}$ and $1:$ the boy who had lived since birth in Stoke, was seen with a characteristic attack of papular urticaria which had developed within 48 hours of his arrival in his grandmother's home in Cardiff. His only previous attack was during an earlier holiday visit to his grandmother at the age of 6 months. His small sister visiting Cardiff for the first time was similarly affected.

Case 73. A girl aged 2 had had extensive lesions for two months. Her skin cleared completely during 10 days in hospital and no new lesions developed. The eruption recurred when she returned home.

Case 87. A boy aged 5 had had papular urticaria continuously from the age of 9 months. He developed no new lesions during the week in hospital but the condition recurred when he returned home.

\section{Discussion}

Lever (1952) contributing to the latest edition of an authoritative textbook on paediatrics writes very briefly on papular urticaria that the cause of the disease is hypersensitivity to insect bites, especially to the bites of bed bugs and fleas'. A review of the literature quickly establishes that the majority of the hypotheses which have at one time or another been put forward can no longer be supported. Study of our own cases is somewhat inconclusive, but we were impressed by the fact that the clinical picture and course of those cases, which seem beyond any possible doubt to be attributable to hypersensitivity to insect bites, differ in no single respect from those cases in which the circumstantial evidence was rather weaker. Although Lever's dogmatism is probably fully justified we feel that on present evidence we can merely claim that sensitivity to insect bites appears to be the cause of the majority and possibly all the cases of papular urticaria we have encountered in South Wales. The possibility that papular urticaria is a syndrome with multiple causes cannot be completely excluded but we found no evidence of any other cause in our own cases and we know of no convincing evidence in the literature that any other cause has ever been reliably established. We would suggest that although the case cannot be considered conclusively proved the physician confronted with a child with papular urticaria is at least under an obligation to employ every available means to exclude a parasitic origin before subjecting the child to any other investigations. Dietary restrictions can no longer be justified.

\section{Treatment}

If papular urticaria be accepted as of parasitic origin a complete revision of conventional treatment naturally follows.

Disinfestation. Careful enquiry will frequently establish the probable nature and source of the infestation and appropriate measures can be adopted to control it. Even when the source of the infestation is not apparent from the history the empirical use of a disinfestation routine can sometimes produce a dramatic and satisfactory cure. Control measures should always be planned after careful consideration of the natural history of the pests. For example, the removal or disinfestation of domestic pets cannot always be relied on as fleas can survive in and around the sleeping quarters and may attack the child, the alternative host, with increased vigour when the natural host is removed. Recent progress in insecticides has been reviewed by Brown (1951) and in a symposium arranged by Buxton (1952). Tentatively, the following simple measures are recommended:-

FLEAS. Treat the animals sleeping quarters with a $5 \%$ D.D.T. spray in kerosene, paying special attention to bedding, floor coverings and crevices. Dust dogs with $5 \%$ D.D.T. powder. For cats 'rotenone' or pyrethrum powder is to be preferred as cats may lick their fur and D.D.T. may prove toxic.

BeD Bugs. Treat floor coverings, floor boards, skirting and furniture with $5 \%$ D.D.T. in kerosene. Residual toxicity is said to persist for nearly $\mathbf{2 0 0}$ days.

Symptomatic Treatment. In the majority of cases if disinfestation is carried out promptly and efficiently calamine lotion and simple sedation with phenobarbitone for a few nights are all that is required. We have not found the antihistamines effective. Where bullous lesions have become secondarily infected antibiotics systemically or locally may be 
indicated. Occasionally when the eruption is exceptionally profuse and of long duration the child may have lost weight as a result of lack of sleep and perhaps unnecessary dietary restrictions. In these cases admission to hospital for a few days may be desirable as it not only establishes the parasitic origin of the eruption beyond doubt but allows time for thorough disinfestation before the child returns home. Eder (1945) claimed that thiamine chloride in a dose of $30 \mathrm{mg}$. daily for three days and $10 \mathrm{mg}$. daily for six weeks gave infants complete protection against flea bites. A limited experience of this procedure has not given encouraging results.

It occasionally happens in cases where there is strong circumstantial evidence incriminating flea or bed bug bites that disinfestation is difficult to carry out effectively or is even quite impracticable. In such cases desensitization deserves consideration but we have had no personal experience of it.

\section{Summary}

After reviewing the literature and investigating a series of 100 cases of papular urticaria we consider that there is strong evidence that most, if not all cases, of papular urticaria are the result of an acquired sensitivity to the bites of certain insects, particularly fleas and bed bugs. Provisionally accepting the validity of this hypothesis recommendations for treatment are outlined.
REFERENCES

Adamson, H. G. (1907). The Skin Affections of Childhoad. p. 255. London.

Barber, H. W. (1914). Practitioner. 93. 820.

Bateman. T. (1813). A Practical Synonsis of Cutaneous Diseases. 2nd edit., p. 13. London.

Blank, H., Shaffer, B., Spencer. M. C. and Marsh, W. C. (1950). Pediatrics, 5. 408.

Botter, A. A. (1952). Dermatologica, Basel. 104, 87.

Bray, G. W. (1937). Recent Advances in Allergy, 3rd edit., p. 365 London.

Brown, A. W. A. (1951). Insect Control by Chemicals. New York and London.

Buxton, P. A. (1952). Trans. rov. Soc. trop. Med., 46. 213.

Cornbleet, T. (1949). Arch. Derm. Syph. Chicago. 60. 1167.

Corson, E. F., Perlman, H. H.. Waltz, A. D. and Ash, R. (1931). Arch. Pediat. 48, 349.

Crocker, H. Radcliffe (1903). Diseases of the Skin. vol. 1. 3rd edit., p. 120 . London.

Dietrich (1938). Mschr. Kinderheilk.. 75. 70.

Eder, H. L. (1945). Arch. Pediat. 62, 300.

Gordon, H. (1933). Lancet. 2. 126.

Gordon, R. M. (1950). Brit. med. J., 2310.

Hallam, R. (1927). Brit. J. Derm., 39. 95 .

(1932). Ibid., 44, 117.

Hamburger, F. and Dietrich (1937). Acta paediat.. ('ppsala, 22, 420

Herms, W. B. (1950). Medical Entomology 4th edit., p. 419. New York.

Hutchinson. J. (1879). Lectures on Clinical Surgery, vol. 1. London. Kinnear, J. (1933). Brit. J. Derm., 45, 65.

Lever, W. F. (1952). The Child in Health and Disease. Ed. C. G Grulee and R. C. Eky. 2nd edit.. p. 986 . London.

Mellanby, K. (1946). Nature Lond.. 158. 554.

Pillsbury, D. M. and Stemberg. T. H. (1937). Amer. J. Dis. Child., 53. 1209

Rockwell, E. M. and Johnson. P. (1952). J. iniest. Derm.. 19, 137.

Sidlick, D. M. and Knowles, F. C. (1922). Amer. J. Dis. Child.. 23. 316.

Shaffer, B. (1950). Arch. Derm. Syph.. Chicago. 62. 259.

- Jacotson, C. and Pori. P. P. (1952). Ann. Allergy, 10. 411.

Tate, B. C. (1935). Archives of Disease in Childhood, 10. 27.

Tomkinson, J. G. (1926). Brit. J. Derm., 38, 431.

Urbach, E. (1938). Derm. Z.. 78. 77.

Walzer, A and Grolnick, M. (1934). J. Allergy. 5. 240.

Winnicott, D. W. (1934). Brit. J. Child. Dis.. 31, 5. 